AMES-HET 94-11

October 1994

\title{
Unitarity And Anomalous Top-Quark Yukawa Couplings
}

\author{
K. Whisnant, Bing-Lin Young, and X. Zhang \\ Department of Physics and Astronomy, \\ Iowa State University, \\ Ames, Iowa 50011
}

\begin{abstract}
Unitarity constraints on anomalous top-Higgs couplings are examined. In the calculation, we considered all $t \bar{t}$ and two-boson channels and obtained the maximal value of the coupled channel amplitude, which sets the unitarity condition. We compare the unitarity constraints with the constraints from electroweak baryogenesis and electric dipole moments derived earlier. Tighter constraints can be obtained by including a $t \bar{t} H H$ contact term which arises from one realization of our effective interaction.
\end{abstract}


Recently the CDF collaboration presented evidence for the top quark with mass $m_{t} \sim 174 \mathrm{GeV}[1]$. Since $m_{t}$ is of order the Fermi scale, the top quark may hold the clue to the physics of the mass generation. In a recent paper[2], we have studied the phenomenology of a non-standard top quark Yukawa coupling. In the notation of Ref. 2, the general interaction of the top quark to the Higgs boson is given by

$$
\mathcal{L}_{t}^{e f f}=\frac{m_{t}}{v} \bar{t}\left[(1+\delta \cos \xi)+i(\delta \sin \xi) \gamma_{5}\right] t H
$$

where $\delta$ is a free parameter, $\xi$ is a $\mathrm{CP}$ phase, and both are zero in the standard model. Such a non-standard top quark Yukawa coupling, $\mathcal{L}_{t}^{e f f}$, is expected to appear in the dynamical electroweak symmetry breaking theories, such as composite Higgs models[3] and (low scale) top quark condensation models[4], and also in extensions of the standard model with fundamental Higgs scalars. In Ref. 2 we sketched a derivation of $\mathcal{L}_{t}^{e f f}$ in a class of the left-right symmetric models[5]. However, the essential motivation for the proposal of $\mathcal{L}_{t}^{e f f}$ is based on the observation that during the electroweak phase transition in the early universe, $\mathcal{L}_{t}^{e f f}$ can help produce the baryon number asymmetry. In Ref. 2, we have shown that for a $\mathrm{CP}$ violation quantity $\delta \sin \xi$ at the level of order $\sim 0.3$ we can understand the matter-antimatter asymmetry of the universe at the weak scale. However, since the electroweak baryogenesis[6] calculations presently available are qualitative and the quantitative results obtained so far are only accurate to within a couple of orders of magnitude, direct measurements of model parameters, e.g., at future colliders, will be needed to test the various models of electroweak baryogenesis. In Ref.[2], we have used electroweak baryogenesis and the electric dipole moments of electron and neutron calculated using $\mathcal{L}_{t}^{e f f}$ to place limits on $\delta$ and $\xi$, and also discussed the possibility of testing $\mathcal{L}_{t}^{e f f}$ directly in the next generation linear colliders. In this paper we will examine the bounds on $\delta$ and $\xi$ from partial-wave unitarity 
constraints.

In the standard model, there is a definite relationship between the fermion mass and its coupling to the Higgs boson, which keeps perturbative unitarity valid at all scales. With non-vanishing $\delta$ and $\xi$, unitarity will be violated at some scale $\sqrt{s}=\Lambda$, where where one expects $\Lambda$ to be the approximate scale at which new particles and interactions appear. The processes affected by the non-standard $H t \bar{t}$ coupling involve the Higgs boson, top quark and weak gauge bosons. First we calculate the amplitudes for $t \bar{t} \rightarrow t \bar{t}$ and $t \bar{t} \rightarrow$ bosons. We will keep only terms of order $G_{F} m_{t} \sqrt{s}$ or $G_{F} m_{t}^{2}$, whichever is larger for a given process. Terms of order $G_{F} m_{W}^{2}, G_{F} m_{b} m_{t}, G_{F} m_{b}^{2}$, or which vanish for large $s$ have little effect on our results and are ignored. We will denote by + or - the helicities of the fermions (written in the order the fermions appear in $t \bar{t} \rightarrow t \bar{t}$ or $t \bar{t} \rightarrow$ bosons). Since we will examine in detail only the $J=0$ partial-wave amplitudes (which gives a stronger constraint than those obtained from higher partial wave amplitudes), only the ++ and -- initial and final state helicity combinations will be discussed. The external gauge bosons involved are longitudinal as they give the leading high energy behavior in the scattering amplitudes.

For the process $t \bar{t} \rightarrow t \bar{t}$ the leading order helicity amplitudes are all constant in $s$. For color-singlet initial and final states there are $s$ - and $t$-channel diagrams involving the photon, $Z$ boson and Higgs boson. To leading order the color-singlet helicity amplitudes are

$$
\begin{aligned}
& T_{++,++}(t \bar{t} \rightarrow t \bar{t})=T_{--,--}^{*}(t \bar{t} \rightarrow t \bar{t})=-3 \sqrt{2} G_{F} m_{t}^{2}\left[1+\left|1+\delta e^{i \xi}\right|^{2}\right] \\
& T_{++,--}(t \bar{t} \rightarrow t \bar{t})=T_{-,+++}^{*}(t \bar{t} \rightarrow t \bar{t})=4 \sqrt{2} G_{F} m_{t}^{2}\left[\left(1+\delta e^{i \xi}\right)^{2}-1\right]
\end{aligned}
$$

Note that the $T_{ \pm \pm, \mp \mp}$ amplitudes in Eq. 2 vanish in the standard model (where $\delta=0)$. For color-octet states only the $t$-channel diagrams contribute and the 
leading order helicity amplitudes are

$$
\tilde{T}_{++,--}(t \bar{t} \rightarrow t \bar{t})=\tilde{T}_{--,++}^{*}(t \bar{t} \rightarrow t \bar{t})=\sqrt{2} G_{F} m_{t}^{2}\left[\left(1+\delta e^{i \xi}\right)^{2}-1\right] .
$$

In Eq. 2 helicity combinations with subleading amplitudes are not listed.

For the process $t \bar{t} \rightarrow W_{L}^{+} W_{L}^{-}$, where $W_{L}$ is a longitudinal $W$ boson, there are $s$-channel diagrams involving the photon, $Z$ boson and Higgs boson, and a $t$-channel diagram with a virtual $b$-quark. In the standard model there are contributions to the amplitudes which are proportional to $s$ and $\sqrt{s}$, but these cancel upon summing over all diagrams. In our case with an effective top-quark Yukawa coupling this cancellation is not assured, and in fact the anomalous part of the Yukawa interaction gives an amplitude proportional to $\sqrt{s}$ in helicity channels with a Higgs boson intermediate state. The leading order color-singlet amplitudes are

$$
T_{++}\left(t \bar{t} \rightarrow W_{L}^{+} W_{L}^{-}\right)=-T_{--}^{*}\left(t \bar{t} \rightarrow W_{L}^{+} W_{L}^{-}\right)=\sqrt{6} G_{F} m_{t} \sqrt{s}\left[\delta e^{i \xi}\right] .
$$

For $t \bar{t} \rightarrow Z_{L} Z_{L}$, there are diagrams with an $s$-channel Higgs boson and a $t$ - and $u$-channel top quark; the leading order color-singlet amplitudes are

$$
T_{++}\left(t \bar{t} \rightarrow Z_{L} Z_{L}\right)=-T_{--}^{*}\left(t \bar{t} \rightarrow Z_{L} Z_{L}\right)=\sqrt{6} G_{F} m_{t} \sqrt{s}\left[\delta e^{i \xi}\right] .
$$

For $t \bar{t} \rightarrow Z_{L} H$, there are diagrams with an $s$-channel $Z$ boson and a $t$ - and $u$ channel top quark; the leading order color-singlet amplitudes are

$$
T_{++}\left(t \bar{t} \rightarrow Z_{L} H\right)=T_{--}^{*}\left(t \bar{t} \rightarrow Z_{L} H\right)=-\sqrt{6} G_{F} m_{t} \sqrt{s}\left[\delta e^{i \xi}\right] .
$$

For $t \bar{t} \rightarrow H H$ the leading color-singlet contribution involving the $t \bar{t} H$ vertex is from an $s$-channel Higgs

$$
T_{++}(t \bar{t} \rightarrow H H)=-T_{--}^{*}(t \bar{t} \rightarrow H H)=-3 \sqrt{6} G_{F} m_{H}^{2} m_{t}\left[1+\delta e^{i \xi}\right] / \sqrt{s} ;
$$


the diagram with a $t$ - or $u$-channel top quark does not contribute to the $J=0$ amplitude. For $m_{H} \approx m_{Z}$ (our region of interest [F.1] ) and $m_{t}=174 \mathrm{GeV}$ the amplitude in Eq. 6 may be neglected, although for $m_{H} \geq \sqrt{m_{t} \sqrt{s}}$ it should be included. Finally, the color-octet amplitudes vanish for $t \bar{t} \rightarrow$ bosons.

With the expressions for the amplitudes in Eqs. 2 to 6, we may now determine the constraints from partial-wave unitarity. The $J=0$ partial-wave amplitude for a process with amplitude $T$ is

$$
a_{0}=\frac{1}{32 \pi} \int_{-1}^{1} T d(\cos \theta) .
$$

Partial-wave unitarity implies that $\left|a_{0}\right|<1$. The most restrictive bound comes from the largest eigenvalue of the coupled channel $T$-matrix. If we write the channels in the order $t_{+} \bar{t}_{+}, t_{-} \bar{t}_{-}, W_{L}^{+} W_{L}^{-}, Z_{L} Z_{L} / \sqrt{2}, Z_{L} H$, and $H H / \sqrt{2}$, then the coupled channel matrix for the color-singlet $J=0$ partial wave is

$$
a_{0}=\frac{\sqrt{2} G_{F} m_{t}^{2}}{16 \pi}\left(\begin{array}{cccccc}
-T_{1} & T_{2} & T_{3} & T_{3} / \sqrt{2} & -T_{3} & -T_{4} / \sqrt{2} \\
T_{2}^{*} & -T_{1} & -T_{3}^{*} & -T_{3}^{*} / \sqrt{2} & -T_{3}^{*} & T_{4}^{*} / \sqrt{2} \\
T_{3}^{*} & -T_{3} & 0 & 0 & 0 & 0 \\
T_{3}^{*} / \sqrt{2} & -T_{3} / \sqrt{2} & 0 & 0 & 0 & 0 \\
-T_{3}^{*} & -T_{3} & 0 & 0 & 0 & 0 \\
-T_{4}^{*} / \sqrt{2} & T_{4} / \sqrt{2} & 0 & 0 & 0 & 0
\end{array}\right)
$$

where

$$
\begin{aligned}
& T_{1}=3\left[1+\left|1+\delta e^{i \xi}\right|^{2}\right], \\
& T_{2}=4\left[1+\delta e^{i \xi}\right]^{2}, \\
& T_{3}=\sqrt{3}\left(\sqrt{s} / m_{t}\right)\left[\delta e^{i \xi}\right], \\
& T_{4}=3 \sqrt{3} m_{H}^{2} /\left(m_{t} \sqrt{s}\right)\left[1+\delta e^{i \xi}\right] .
\end{aligned}
$$

In Eq. 8 we must also include all channels of the type bosons $\rightarrow$ bosons. The $t$ quark Yukawa coupling does not contribute to these processes at the tree level, so [F.1] This is required by baryogenesis to avoid washout of the asymmetry[8]. 
these amplitudes assume their standard model values, which are of order $G_{F} m_{H}^{2}$. Since in our scenario $m_{H} \approx m_{Z}$, this is small compared to $G_{F} m_{t} \sqrt{s}$ and $G_{F} m_{t}^{2}$, and we can set the purely bosonic amplitudes to zero as indicated in Eq. 8. We have checked that the amplitudes for these channels are at most a few percent of the dominant amplitudes in the coupled-channel matrix for the values of the parameters we are considering, which justifies this approximation. Similarly the contribution of $T_{4}$ in Eq. 6 is small, and therefore the last row and column in Eq. 8 can be neglected (for a different case with a non-negligible $T_{4}$, see below).

The characteristic equation for the roots of the remaining $5 \times 5$ matrix is easily found. After removing a trivial zero root, the root with the largest magnitude may be found numerically. The corresponding upper bound on $\delta$ as a function of $\xi$ is shown as the dashed curve in Fig. 1, assuming unitarity is preserved up to $\sqrt{s}=1 \mathrm{TeV}$. Also shown for comparison are the approximate lower bound from electroweak baryogenesis and the upper bound from the neutron electric dipole moment [2]. We should point out that the constraints on $\delta$ and $\xi$ depend on the $\sqrt{s}$ at which we assume that unitarity is saturated. We have also checked that if the magnitude of the anomalous coupling is comparable to the standard model coupling, the scale of the new physics should be no more than a few $\mathrm{TeV}[9]$.

Before concluding, we should also point out that a sizable $T_{4}$ can be generated even for $m_{H} \simeq m_{Z}$ if higher dimension operators are considered. Such operators can arise quite naturally in a linearly realized $S U(2)_{L} \times U(1)_{Y}$ effective Lagrangian. For example, the operator[2]

$$
\mathcal{O}^{t}=\delta e^{i \xi}\left(\frac{\Phi^{\dagger} \Phi}{v^{2}}-\frac{1}{2}\right) \overline{\left(\begin{array}{c}
t_{L} \\
b_{L}
\end{array}\right)} \tilde{\Phi} t_{R}+\text { h.c. }
$$

where $\Phi$ is the complex standard model doublet scalar and $\tilde{\Phi}=i \sigma_{2} \Phi^{*}$, will generate $t \bar{t} H H$ and $t \bar{t} H H H$ contact terms in addition to the anomalous part of the Yukawa coupling in Eq. 1. Since in the broken phase $\Phi \rightarrow(H+v) / \sqrt{2}$, the ratio 
of the $t \bar{t} H H$ to $t \bar{t} H$ coupling strengths in Eq. 10 is fixed to be $3 / 2 v$. The contribution from the contact term $t \bar{t} H H$ then dominates the color-singlet $t \bar{t} \rightarrow H H$ amplitude; we find the new amplitude

$$
T_{4}=T_{++}(t \bar{t} \rightarrow H H)=-T_{--}^{*}(t \bar{t} \rightarrow H H)=6 T_{3} .
$$

Now $T_{4}$ is proportional to $G_{F} m_{t} \sqrt{s}$ and the full $6 \times 6$ matrix in Eq. 8 must be used. The unitarity limit derived from the largest eigenvalue in this case is shown as the solid curve in Fig. 1. We note that in general for an effective lagrangian with non-linear realization of $S U(2)_{L} \times U(1)_{Y}$ (where $H$ is considered a scalar singlet) there is an independent free parameter associated with each contact term, which makes it impossible to improve the unitarity bounds on the anomalous Yukawa coupling by considering the effect of the contact terms on the scattering amplitude. In this case the bound found by setting $T_{4}=0$ will be the upper limit.

In conclusion, we have examined the unitarity constraints on anomalous topHiggs couplings. Our results for $\Lambda=1 \mathrm{TeV}$, where $\Lambda$ is the mass scale of the new physics which produces such an anomalous top-Higgs interaction, are summarized in Fig.1. From the figure we see that $\delta$, the magnitude of the anomalous top Yukawa coupling, should lie in the range $0.3-3.5$ in order to satisfy all of the constraints. One can also turn this requirement around. We have checked that for $\delta \sin \xi \sim 0.3$, as implied by electroweak baryogenesis[2], unitarity requires that $\Lambda$ is less than about $5 \mathrm{TeV}$. Since this new physics scale is not too far from the Fermi scale, one might expect that new physics effects will not only show up as a modified Yukawa interaction (Eq. 1), but also in anomalous couplings of the top quark to the gauge bosons, which will be examined later.

This work is supported in part by the Office of High Energy and Nuclear Physics of the U.S. Department of Energy (Grant No. DE-FG02-94ER40817). 


\section{Figure Captions}

[Fig.1] Bounds on a non-standard top-quark Yukawa coupling in terms of the parameters $\delta$ and $\xi$. The solid curve is the upper bound from unitarity using the $t \bar{t} H$ and $t \bar{t} H H$ anomalous interactions and the dashed curve is the bound from the $t \bar{t} H$ interaction alone, for $\Lambda=1 \mathrm{TeV}$. The dotted curve is an approximate lower bound from electroweak baryogenesis and the dash-dotted curve is an upper bound from the experimental limit on the electric dipole moment of the neutron, taken from Ref. 2. 


\section{References}

[1] CDF Collaboration, F. Abe et al, FERMILAB-PUB-94/097-E, 1994.

[2] X. Zhang, S. Lee, K. Whisnant and B.-L. Young, "Phenomenology of a non-standard top quark Yukawa coupling," Iowa State University Preprint, AMES-HET-94-05, June 1994 (to appear in Phys. Rev. D).

[3] D.B. Kaplan and H. Georgi, Phys. Lett. B136, 183 (1984); D.B. Kaplan, S. Dimopoulos and H. Georgi, Phys. Lett. B136, 187 (1984); H. Georgi, D.B. Kaplan and P. Galison, Phys. Lett. B143, 152 (1985); H. Georgi and D.B. Kaplan, Phys. Lett. B145, 216 (1984); M.J. Dugan, H. Georgi and D.B. Kaplan, Nucl. Phys. B254, 299 (1985); V. Koulovassilopoulos and R.S. Chivukula, BUHEP-93-30 (hep-ph/9312317), Dec. 20, 1993.

[4] C.T. Hill, Phys. Lett. B266, 419 (1991).

[5] J.C. Pati and A. Salam, Phys. Rev. D10, 275 (1974); R.N. Mohapatra and J.C. Pati, ibid. D11, 566 (1975); D11, 2558 (1975); Senjanovic and R.N. Mohapatra, ibid. D12, 1502 (1975).

[6] For a review, see, A. Cohen, D. Kaplan and A. Nelson, Ann. Rev. Nucl. Part. Sci. 43, 27 (1993); on recent development on spontaneous baryogenesis, see, A.G. Cohen, D.B. Kaplan, A.E. Nelson, Phys. Lett. B336, 41 (1994), and references therein.

[7] For a discussion on unitarity bounds on the Higgs-boson and top-quark masses, see, W. Marciano, G. Valencia, and S. Willenbrock, Phys. Rev. D40, 1725 (1989), and references therein; for a discussion on unitarity bounds on anoma-

lous gauge boson couplings, see, G. Gounaris, J. Layssac, J. Paschalis and F. Renard, PM/94-28, THES-TP 94/08, August 1994.

[8] X. Zhang, B.-L. Young, Phys. Rev. D49, 563 (1994); and references therein. 
[9] M. Golden, Harvard University Preprint, HUTP-94/A020, July 1994, hep$\mathrm{ph} / 9408272$. 